\title{
Comparison of the influence of stimuli color on Steady-State Visual Evoked Potentials
}

\author{
Richard Junior Manuel Godinez Tello*, Sandra Mara Torres Müller, André Ferreira, \\ Teodiano Freire Bastos
}

\begin{abstract}
Introduction: The main idea of a traditional Steady State Visually Evoked Potentials (SSVEP)-BCI is the activation of commands through gaze control. For this purpose, the retina of the eye is excited by a stimulus at a certain frequency. Several studies have shown effects related to different kind of stimuli, frequencies, window lengths, techniques of feature extraction and even classification. So far, none of the previous studies has performed a comparison of performance of stimuli colors through LED technology. This study addresses precisely this important aspect and would be a great contribution to the topic of SSVEP-BCIs. Additionally, the performance of different colors at different frequencies and the visual comfort were evaluated in each case. Methods: LEDs of four different colors (red, green, blue and yellow) flickering at four distinct frequencies $(8,11,13$ and $15 \mathrm{~Hz})$ were used. Twenty subjects were distributed in two groups performing different protocols. Multivariate Synchronization Index (MSI) was the technique adopted as feature extractor. Results: The accuracy was gradually enhanced with the increase of the time window. From our observations, the red color provides, in most frequencies, both highest rates of accuracy and Information Transfer Rate (ITR) for detection of SSVEP. Conclusion: Although the red color has presented higher ITR, this color was turned in the less comfortable one and can even elicit epileptic responses according to the literature. For this reason, the green color is suggested as the best choice according to the proposed rules. In addition, this color has shown to be safe and accurate for an SSVEP-BCI.
\end{abstract}

Keywords: Brain Computer Interface, color, EEG, LED, SSVEP.

\section{Introduction}

The human eye is composed of three color-sensitive cone-cell types (red, green and blue). According to studies performed by W. D. Wright (Gregory, 1997), these three cone types have different responses for different stimulus wavelengths. Among the three cone types, the red-cone presents the best response followed closely by the green-cone, and with the blue one having the lowest response.

Experiments with visual stimuli have been performed about decades ago (Bieger and Garcia-Molina, 2010; Regan, 1966). These stimuli produce a stable Visual Evoked Potential (VEP) termed as "Steady-State" Visually Evoked Potentials (SSVEPs) of the human visual system. The amplitude and phase of the SSVEP are highly sensitive to stimulus parameters, such as color, luminance, repetition rate, contrast (modulation depth), and frequency.

On the other hand, flicker stimuli can elicit epileptic responses to certain luminance or chromaticity. Higher luminance can induce a higher risk of epilepsy (Vialatte et al., 2010) and the chromaticity of a visual stimulus has a strong impact on the human eye response in case of combination of colors (Drew et al., 2001). Red/blue and green/blue combinations have the strongest effect on pupil constriction and they can produce seizure attacks (Drew et al., 2001). Regarding frequency dependency, repetitive visual stimuli modulated at certain frequencies can also provoke epileptic seizures. According to Drew et al. (2001), lower frequency flickers generally produce more powerful constrictions, with color-dependence of flickers most visible between 3 and $6 \mathrm{~Hz}$. In other study, Fisher et al. (2005) show that flash and pattern reversal stimuli can provoke epileptic seizures especially in the $15-25 \mathrm{~Hz}$ range, but for some people, the upper limit of sensitivity can be as high as $65 \mathrm{~Hz}$ (Fisher et al., 2005).

There are some studies about the effects of colors on both SSVEP response and measurement of Information Transfer Rate (ITR), plus a qualitative feedback of comfort provided by the volunteers (Bieger et al., 2010; Cao et al., 2012; Regan, 1966). The term ITR is a quantitative parameter of measurement of performance expressed in quantity of bits per 
minute. In 1966, a study with red, yellow, and blue stimuli was performed (Regan, 1966), however, these flashing lights were not from LEDs (Light Emitting Diodes) technology. According to that study, red color elicited the strongest response when modulated at approximately $11 \mathrm{~Hz}$, and the response dropped dramatically at neighboring frequencies. Blue stimuli elicited a slightly weaker response, around $13 \mathrm{~Hz}$, and the SSVEP strength elicited by the yellow color was the lowest in that study.

Other study (Bieger et al., 2010) was performed in order to evaluate the refresh rate, environmental illumination, contrast, color, spatial frequency and size of visual stimuli. Single graphics stimuli (red, white, blue, green), color combination and reversal patterns (checkerboards) were also analyzed. That work concluded that the white color got the highest value of ITR $(\approx 55 \mathrm{bits} / \mathrm{min}$ ), although reporting the lower level of comfort by users. The blue color presented the highest level of comfort, with ITR of $\approx 32 \mathrm{bits} / \mathrm{min}$. The red and green colors had similar level of comfort, with ITR of 33 and $38 \mathrm{bits} / \mathrm{min}$, respectively. The stimuli were displayed on a LCD (Liquid Crystal Display) screen in that study.

The work performed in Cao et al. (2012) is similar to a part of the work developed in Bieger et al. (2010). It means that the stimuli were generated in a LCD screen with a black background and single stimulus. The stimulus colors were red, white, blue, green and gray. That work also concluded that the white color achieved the highest ITR, followed by gray, red, green and blue colors. The average ITR was $32.3 \mathrm{bits} / \mathrm{min}$.

Although the ITR can evaluate the effectiveness of each color, it is dependent on the signal processing technique used. However, there is no study about the effect of stimulus color on the SSVEP amplitude using LEDs as visual stimulator. Thus, evaluating the SSVEP amplitude using LEDs is a must for now since this technology of stimulation remains of high usability due to its low cost, low power, portability and flexibility. A comparative study of technologies for visual stimuli (LCD vs LED) was reported in Wu et al. (2008), which concludes that the SSVEP response elicited by LEDs is higher than that of a LCD screen. However, that study did not present any kind of comparison about stimulus colors.

In this present study, four flickering stimuli with different LED colors are used. The analyzed colors are red, green, blue and yellow. There is no color combination (for safety reasons), and four frequency values are used for the stimuli according to our previous work (Tello et al., 2014a). This study also evaluates the performance of such specific colors at different frequencies. A qualitative score of the degree of comfort is also achieved. The results found in this work are valuable to improve the performance of a Brain-Computer Interface (BCI) based on SSVEP. So far, none of the previous studies did comparison of the performance of stimuli's colors using LED technology, which is done in this work.

\section{Methods}

\section{Subjects}

Twenty subjects (seventeen males and three females), with ages from 21 to 36 years old, were recruited to participate in this study (average age: 27.9; Standard Deviation (SD): 3.7). The research was carried out in compliance with Helsinki declaration, and the experiments were performed according to the rules of the ethics committee of UFES/Brazil, under registration number CEP-048/08. The subjects were distributed into two experiments. The first group (Group 1) with ten volunteers: s1, s2, s3, s4, s5, s6, s7, s8, s9 and s10; and the second one (Group 2) with ten volunteers remaining: s11, s12, s13, s14, s15, s16, s17, s18, s19 and s20; both using different protocols, which will be discussed in subsequent lines.

All measurements were noninvasive and the subjects were free to withdraw at any time without any penalty. Previously, a selection of volunteers was performed and topics related to precautions as visual problems, headaches, family history with epilepsy and problems related to brain damage were consulted. The participants did not report any problems. No one had previous experience in using a BCI.

\section{EEG recording}

A BrainNet-36 equipment was the device used for electroencephalogram (EEG) acquisition through a cap with $\mathrm{Ag}-\mathrm{AgCl}$ wet equidistant electrodes. This device is manufactured for clinical purposes by Lynx Tecnologia Ltd (Brazil). The data were processed on a personal computer with a $2.2 \mathrm{GHz}$ Core 2 Duo processor.

For the development of this study, EEG signals from 12 channels, placed on the occipital part of the cortex with the reference electrode at the left ear lobe were recorded at 600 samples/s, with 1 to $100 \mathrm{~Hz}$ pass-band filter. The ground electrode was placed on the forehead. The EEG electrode placements were based on the International 10-20 System. The electrodes used were: P7, $\mathrm{PO} 7, \mathrm{PO} 5, \mathrm{PO} 3, \mathrm{PO}$, $\mathrm{PO} 4, \mathrm{PO} 6, \mathrm{PO}$, P8, $\mathrm{O} 1, \mathrm{O} 2$ and $\mathrm{Oz}$. The EEG recordings in all subjects were executed by the same technician to minimize operator errors.

It is worth to comment that for both SSVEP response and classification studies, only $\mathrm{O} 1, \mathrm{O} 2$ and 
$\mathrm{O} 3$ electrodes were selected after the application of a Common Average Reference (CAR) spatial filter, which is based on studies that suggest that the highest values of energy for SSVEP detection are located on the occipital area of the cortex (Di Russo et al., 2007; Krolak-Salmon et al., 2003; Pastor et al., 2003; Sammer et al., 2005; Zhang et al., 2006). Thus, the twelve aforementioned electrodes were used at the initial stage only for application of the CAR spatial filter. According to our observations, the application of this spatial filter to the twelve electrodes actually improves the classification performance when selecting $\mathrm{O} 1, \mathrm{O} 2$ and $\mathrm{Oz}$ electrodes.

\section{Stimulation unit (SU)}

A coupling structure of four small boxes $(4 \mathrm{~cm} \times 4 \mathrm{~cm} \times 4 \mathrm{~cm})$ containing a LED in each one and covered with thin white paper diffusers was mounted. The diffused paper is also known as wax paper or paraffin paper and has the following properties: semi-transparent, completely smooth and moisture-proof. Figure 1a shows the block diagram of the acquisition and stimulation system.

The stimuli are generated by a microcontroller (PIC18F4550, Microchip Technology Inc., USA) with $50 / 50 \%$ on-off duty cycles. LEDs of $5 \mathrm{~mm}$ (part number: NCM: 85414022) of four different colors (yellow, red, green, blue) and same luminous intensity $(10,000 \mathrm{mcd})$ were used. Luminous intensity values of each LED were controlled by the microcontroller outputs (same port), guaranteeing, in this way, the same levels of intensity luminosity. In addition, these values were measured through a light meter (ICEL MANAUS, model LD-590).

The flickering frequencies were $8.0 \mathrm{~Hz}, 11.0 \mathrm{~Hz}$, 13.0 Hz and $15.0 \mathrm{~Hz}$, which were used by the four colors of LEDs. These frequencies were chosen due to: 1) our previous studies (Tello et al., 2014a; 2014b; 2014c) have shown that these generate strongest SSVEP responses; 2) safety recommendations specified in Fisher et al. (2005); 3) studies conducted by Pastor et al. (2003) about the relationship between visual stimuli and SSVEP-evoked amplitudes recommend these frequencies; 4) studies conducted by Herrmann (2001) showing peaks of SSVEPs at $\sim 15 \mathrm{~Hz}$.

\section{Experimental procedure}

The experiments were performed in offline mode. The participants were asked to observe the stimuli during $320 \mathrm{~s}$. The stimuli consisted of sixteen sequences: four sequences for each color with four different frequencies. Each stimulus sequence lasted $15 \mathrm{~s}$, followed by a $5 \mathrm{~s}$ of pause (rest), during whose time the EEG signals were recorded.
The two groups of volunteers (Group 1 and 2) followed different protocols in order to validate the results. In addition, Figure 1a shows a block called synchronization signal, which means a logical level from an algorithm developed in Matlab that generates a specific protocol for each group of participants. In that algorithm, volunteers of Group 1 underwent certain stimuli, which are graphically detailed in Figure 1b; this protocol is called as "ordered", because colors and frequencies followed a determined order. On the other hand, the volunteers of Group 2 were stimulated in a randomic way in both frequency and color, calling this protocol as "randomic" (see Figure 1c).

A structure moved the boxes that contained the LEDs during each sequence of acquisition aiming at centralizing the flashing stimulus and keeping it completely still in front of the volunteer in order to standardize of the protocol. The experiments were conducted in a quiet and dim room with illuminance of 300 lux measured through the light meter. Then, the volunteers sat on a comfortable chair, in front of the stimulator system, $60 \mathrm{~cm}$ far from this. The background plane was a black wall located very close and behind the stimuli.

\section{EEG pre-processing and SSVEP response analysis}

A Common Average Reference (CAR) spatial filter was applied to the EEG signals corresponding to each stimulation frequency and for each color in order to reduce the correlation between channels originated by external noise. The CAR filter was computed according to Equation 1 (McFarland et al., 1997):

$V_{i}^{C A R}=V_{i}^{E R}-\frac{1}{n} \sum_{j=1}^{n} V_{j}^{E R}$,

where $V_{i}^{E R}$ is the potential between the $i$ th electrode and the reference, and $\mathrm{n}$ is the number of electrodes used (in our case, twelve).

After that, just the resultant signals from $\mathrm{O} 1, \mathrm{O} 2$ and $\mathrm{Oz}$ electrodes were selected, and a 5th-order elliptic band-pass filter between 3-60 Hz was applied. Since the elliptic filter has a transition band narrower than other filters and as harmonic components are very close, this type of filter was chosen as the more suitable. This filter has $1 \mathrm{~dB}$ of peak-to-peak pass-band ripple and $15 \mathrm{~dB}$ of stop-band attenuation down the pass-band value.

The SSVEP amplitude response is obtained through PSDA (Power Spectral Density Analysis) method or Fourier Power Analysis (Wang et al., 2006) with Hamming window. SSVEPs response curves were calculated by the following formula: 
$P_{\text {Norm }}(f)=\frac{\bar{P}_{f}}{\max _{f} \bar{P}_{f}^{V S}}$,

where $P_{\text {Norm }}(f)$ is a normalized amplitude value obtained by the relation between the average value $\left(\bar{P}_{f}\right)$ among all subjects for each case (group and frequency), and $\max _{f} \bar{P}_{f}^{V \mathrm{~S}}$ denotes the maximum average amplitude value obtained from all cases of visual stimulation $(V \mathrm{~S})$ analyzed between groups.

Such as aforementioned, the data from twelve EEG channels were segmented and windowed. The window lengths used were 1, 2, 4 and $6 \mathrm{~s}$; each

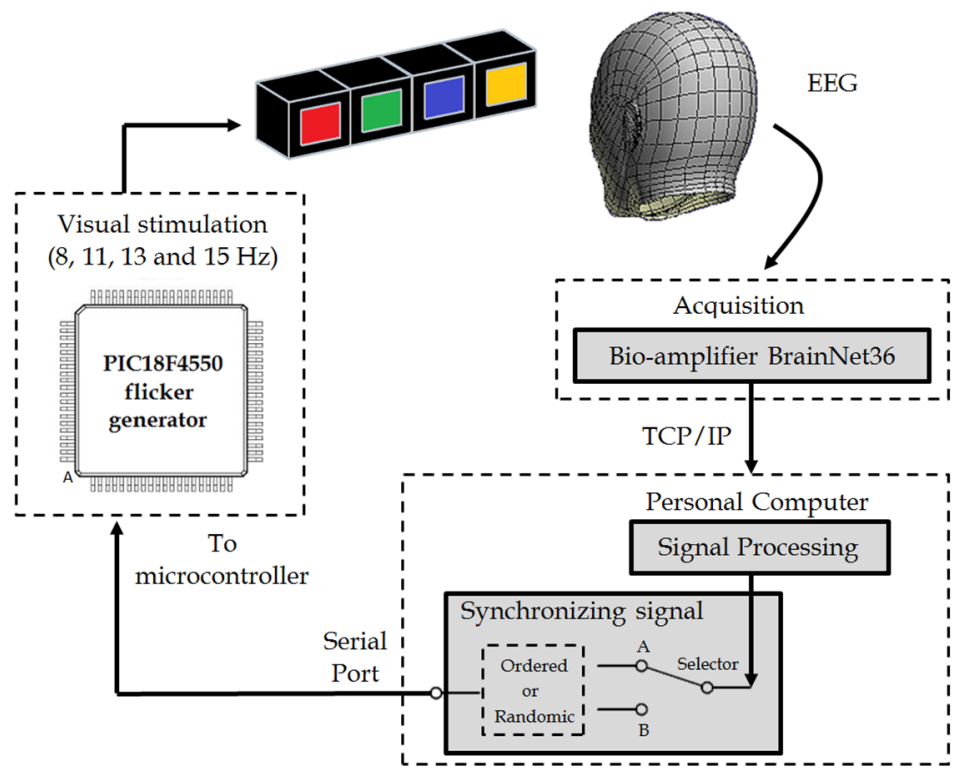

(a)

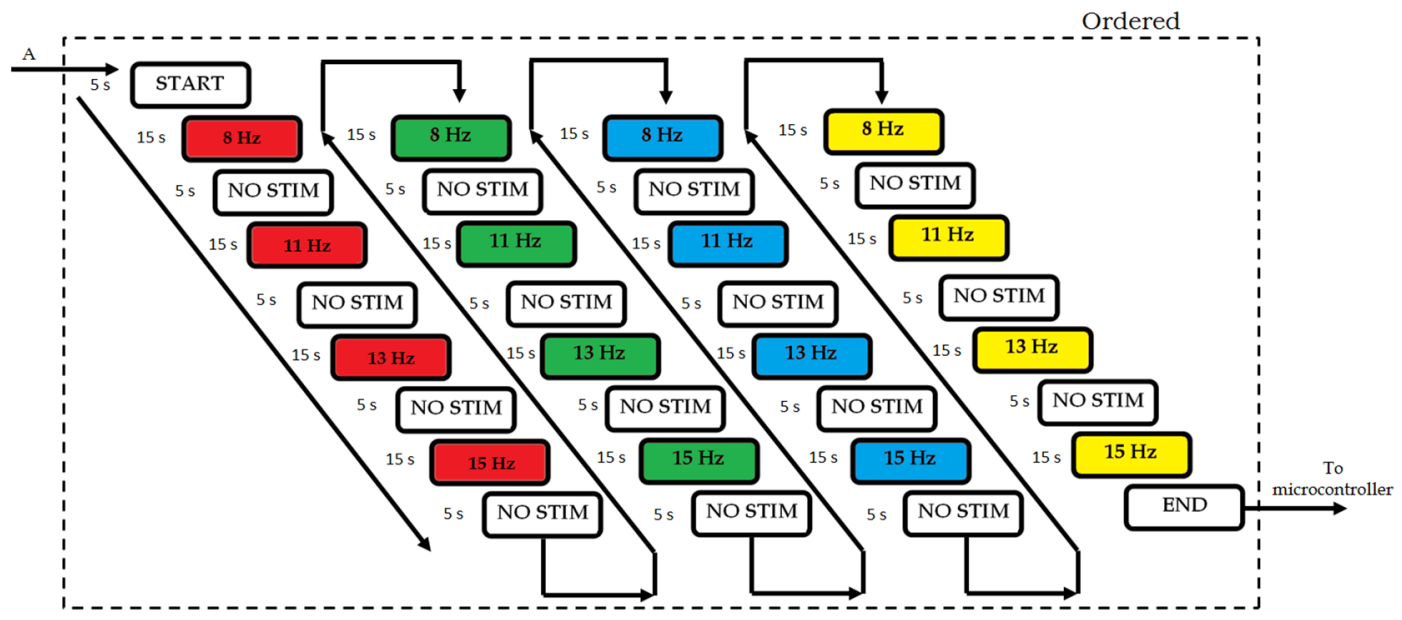

(b)

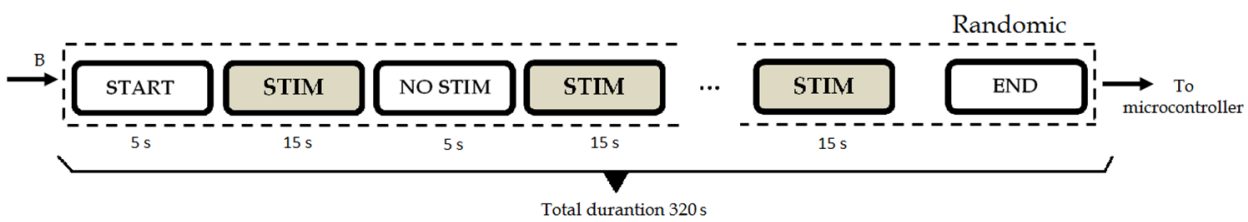

(c)

Figure 1. (a) Block diagram of the system; (b) Protocol used by the volunteers of Group 1 called "ordered"; (c) Protocol used by the volunteers of Group 2 called "randomic". Note that both protocols were performed with the same time duration of 320s. 
one with an overlapping of $50 \%$. Signals from O1, $\mathrm{O} 2$ and $\mathrm{Oz}$ channels were selected after the process of CAR spatial filtering. After that, a 5th-order elliptic band-pass filter between 3-60 Hz was applied and the resultant signals were used in the feature extractor and classification steps.

\section{Feature extraction and classification}

Multivariate Synchronization Index (MSI) is a novel method to estimate the synchronization between the actual mixed signals and the reference signals as a potential index for recognizing the stimulus frequency. Zhang et al. (2014) have proposed the use of a $S$ -estimator as index, which is based on the entropy of the normalized eigenvalues of the correlation matrix of multivariate signals.

Mathematically, this method assumes that $\mathrm{X}$ is a matrix of size $N \times M$ whose $N$ represents discrete-time EEG signal segments and $M$ represents the channels from the occipital electrodes $(\mathrm{O} 1, \mathrm{O} 2$ and $\mathrm{Oz}$ ). On the other hand, $\mathrm{Y}_{i}$ consists of a "Fourier series" of stimulus signals.

Let $f_{i}$ denote the visual stimulus frequency in $\mathrm{Hz}$, where $i=1\left(f_{1}=8 \mathrm{~Hz}\right), 2\left(f_{2}=11 \mathrm{~Hz}\right)$, $3\left(f_{3}=13 \mathrm{~Hz}\right)$ and $4\left(f_{4}=15 \mathrm{~Hz}\right)$ denotes the target or class of our visual stimuli. Then, the total $H$ harmonic sine vectors $s_{1}^{i}, s_{2}^{i}, \ldots, s_{H}^{i}$ and cosine vectors $c_{1}^{i}, c_{2}^{i}, \ldots, c_{H}^{i}$ for frequency $f_{i}$, all of length $M$, can be constructed as

$s_{j}^{i}=\left[s_{j, 1} s_{j, 2} \ldots s_{j, M}\right]^{T} \quad c_{j}^{i}=\left[c_{j, 1} c_{j, 2} \ldots c_{j, M}\right]^{T}$

for $j=1,2, \ldots, H$, where

$$
s_{j, r}=\sin \left(2 \pi j r f_{i} / f_{s}\right) c_{j, r}=\cos \left(2 \pi j r f_{i} / f_{s}\right),
$$

for $r=[0: N-1]$, and $f_{s}=600 \mathrm{~Hz}$ is the sampling frequency used for the acquisition of EEG signals. The reference matrix $\mathrm{Y}_{i}$, of size $2 H \times M$, corresponds to the stimulus frequency $f_{i}$, which can be constructed as

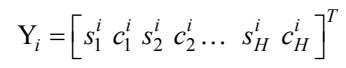

In our case, we have considered the fundamental frequency (considered as the first harmonic) and two multiples (harmonics) as the simulated frequency generator, i. e. $H=3$. Autocorrelation matrices $\mathrm{C}_{11}$ and $\mathrm{C}_{22}$ for $\mathrm{X}$ and $\mathrm{Y}_{i}$, respectively, and cross-correlation matrices $\mathrm{C}_{12}$ and $\mathrm{C}_{21}$ were changed from the original version (Zhang et al., 2014) due to its inconsistency in the dimensions of each component for the formation of the correlation matrix $\mathrm{C}^{i}$. The efficiency of our algorithms has been demonstrated in several of our works (Tello et al., 2014a; 2014b; 2014c; 2015). Thus, the following equations are proposed:

$$
\begin{aligned}
& \mathrm{C}_{11}=(1 / M) \cdot \mathrm{XX}^{T} \\
& \mathrm{C}_{22}=(1 / M) \cdot \mathrm{Y}_{i} \mathrm{Y}_{i}^{T} \\
& \mathrm{C}_{12}=(1 / M) \cdot \mathrm{XY}_{i}^{T} \\
& \mathrm{C}_{21}=(1 / M) \cdot \mathrm{Y}_{i} \mathrm{X}^{T}
\end{aligned}
$$

A correlation matrix $\mathrm{C}^{i}$ can be constructed as

$$
C^{i}=\left[\begin{array}{ll}
C_{11} & C_{12} \\
C_{21} & C_{22}
\end{array}\right]
$$

The internal correlation structure of $\mathrm{X}$ and $\mathrm{Y}_{i}$ contained in the matrices $\mathrm{C}_{11}$ and $\mathrm{C}_{22}$, respectively, is irrelevant for the detection of stimulus frequency (Carmeli et al., 2005). It can be removed by constructing a linear transformation matrix

$\mathrm{U}=\left[\begin{array}{cc}\mathrm{C}_{11}^{-1 / 2} & 0 \\ 0 & \mathrm{C}_{22}{ }^{-1 / 2}\end{array}\right]$

so that $\mathrm{C}_{11}{ }^{1 / 2} \mathrm{C}_{11}{ }^{1 / 2}=\mathrm{C}_{11}, \mathrm{C}_{22}{ }^{1 / 2} \mathrm{C}_{22}{ }^{1 / 2}=\mathrm{C}_{22}$ and, by applying the transformation $\tilde{C}^{i}=\mathrm{UCU}$, it results in a transformed correlation matrix

$$
\tilde{C}^{i}=\left[\begin{array}{cc}
I_{N \times N} & \mathrm{C}_{11}{ }^{-1 / 2} \mathrm{C}_{12} \mathrm{C}_{22}{ }^{-1 / 2} \\
\mathrm{C}_{22}{ }^{-1 / 2} \mathrm{C}_{21} \mathrm{C}_{11}{ }^{-1 / 2} & I_{2 H \times 2 H}
\end{array}\right]
$$

of size $P \times P$, where $P=N+2 H$. The eigenvalues of $\lambda_{1}^{i}, \lambda_{2}^{i}, \ldots, \lambda_{P}^{i} \tilde{C}^{i}$, normalized as $\tilde{\lambda}_{m}^{i}=\lambda_{m}^{i} / \sum_{m=1}^{P} \lambda_{m}^{i}$ for $m=1,2, \ldots, P$, can be used to evaluate the synchronization index $S_{i}$ for matrix $\mathrm{Y}_{i}$, as in Zhang et al. (2014):

$$
S_{i}=1+\frac{\sum_{m=1}^{P} \tilde{\lambda}_{m}^{i} \log \left(\tilde{\lambda}_{m}^{i}\right)}{\log (P)}
$$

Then, $i$ indices and their respective classes $\left(S_{1}, S_{2}, S_{3}, S_{4}\right)$ were obtained. Finally, the class was obtained through a criterion of maxima.

$S=\max _{1 \leq i \leq 4} S_{i}$

\section{Results}

Figure 2 shows the SSVEP response curves for each group of volunteers. From these responses curves, Table 1 shows the maximum peaks values for each group evaluated.

The accuracy rate and Information Transfer Rate (ITR) were computed. In order to calculate this last 
Group 1

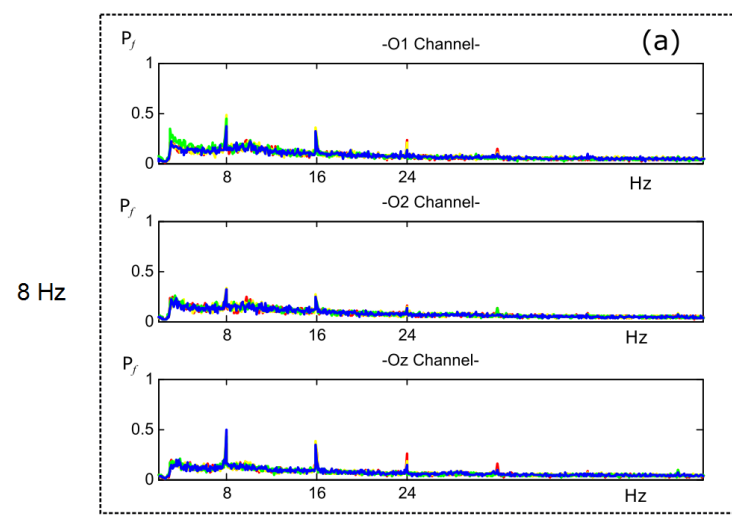

11 нस
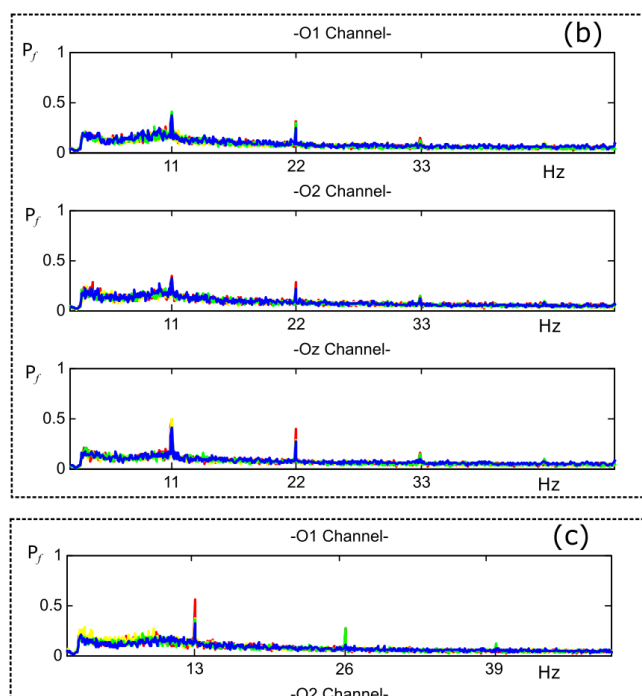

$13 \mathrm{~Hz}$
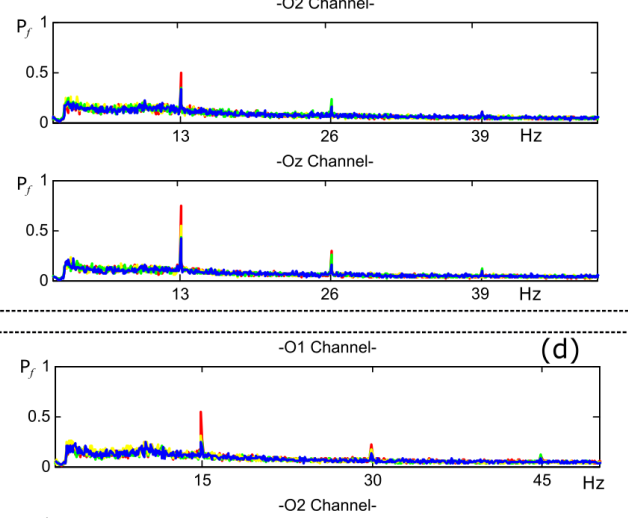

$15 \mathrm{~Hz}$
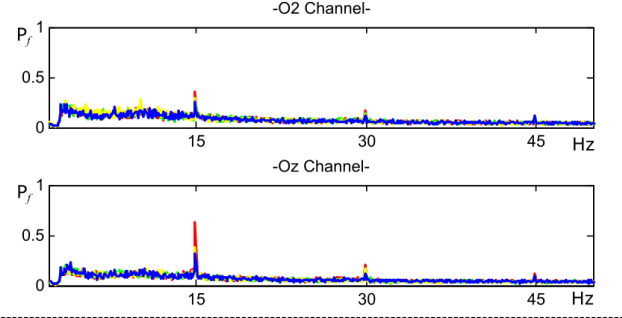

\section{Group 2}
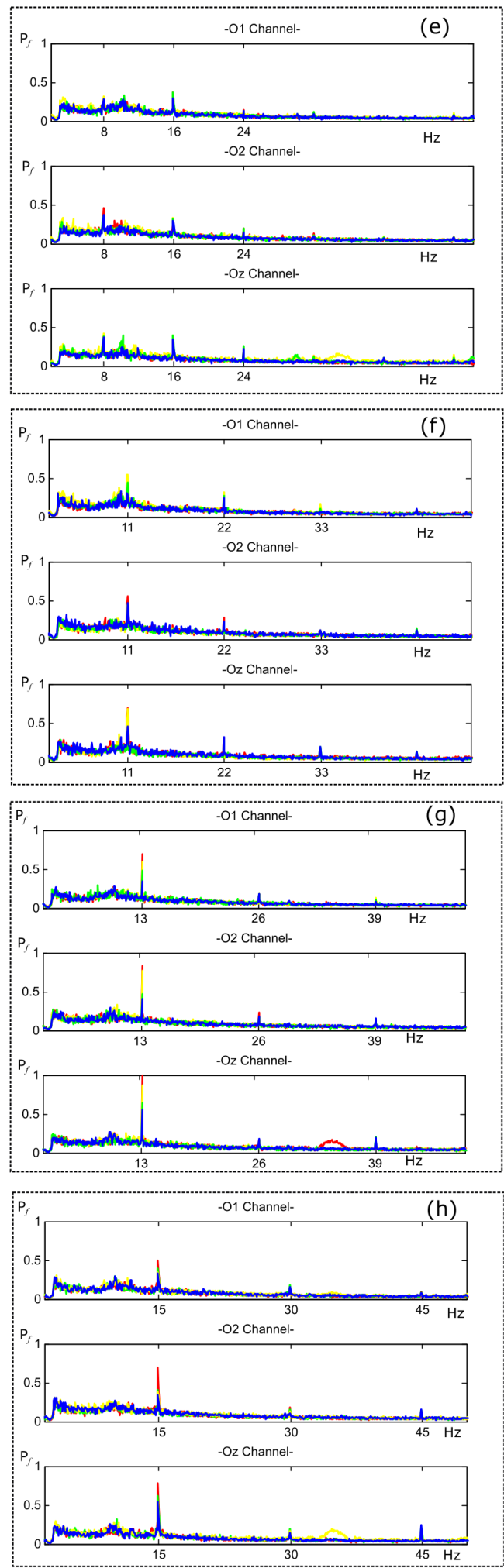

Figure 2. Normalized SSVEP response curves for channels O1, O2 and Oz from average results of volunteers of Group 1: ((a) $8 \mathrm{~Hz}$, (b) $11 \mathrm{~Hz}$ (c) $13 \mathrm{~Hz}$ and (d) $15 \mathrm{~Hz}$ ); normalized SSVEP response curves for channels O1, O2 and Oz from volunteers of Group 2: ((e) $8 \mathrm{~Hz}$, (f) $11 \mathrm{~Hz}$, (g) $13 \mathrm{~Hz}$ and (h) $15 \mathrm{~Hz}$ ). The color of the curves indicates the analyzed color. 
parameter, firstly a Command Transfer Interval (CTI) was defined according to the following ratio: number of total outputs (Ntotal) by each time interval (Ttotal), i.e., Ntotal/Ttotal. Thus, the values of (CTI) are the windows lengths or also called time windows $(\mathrm{TW}=1,2,4$ and $6 \mathrm{~s})$ for each case. Be understood that each packet of window size means a trial to evaluate. Then, the Shannon's Information Transfer Rate (ITR) (Vialatte et al., 2010), considered the most common measurement to assess the performance of a $\mathrm{BCI}$ system was used, which is defined by Equation 15 and 16 (Kelly et al., 2005a):

$\frac{\text { Bits }}{\text { Command }}=\log _{2} A+A \log _{2} A+(1-A) \log _{2}\left(\frac{1-A}{K-1}\right)$,

$\mathrm{ITR}=\frac{\text { Bits }}{\text { Command }} \times \frac{60}{\mathrm{CTI}}$,

where $K$ is the total number of stimuli and $A$ is the accuracy rate. In addition to the bit rate, after the experiments the volunteers were asked to give an opinion on the degree of comfort of each color of stimuli on a 7-point scale, ranging from low to high comfort, based on their subjective observations.

Table 2 presents these scores provided by the volunteers. A statistical analysis was implemented using a nonparametric method of two-way analysis named Friedman's test with the purpose of finding out any effect for the level of comfort of the colors for each groups of volunteers (Group 1 and Group 2). The results showed a statistic significance with $p=1.8949 \times 10-5$ using alpha $=0.05$.

Figure 3 shows the average SSVEP recognition accuracy of Group 1 and 2 derived by the MSI method, respectively, with different time windows (TW $=1$, 2, 4 and $6 \mathrm{~s})$ and frequencies $(8,11,13$ and $15 \mathrm{~Hz})$.

Based on the results of Figure 3, the accuracy was gradually enhanced with the increase of TW for all frequencies used and group of volunteers. The TW of $4 \mathrm{~s}$ was adopted as parameter of classification since the results were good and very similar compared to the TW of $6 \mathrm{~s}$ in most of the cases analyzed.

Figure 4 shows the classification results in a box plot representation corresponding to the different

Table 1. Maximum peaks values for each normalized amplitude response. The highlights numbers indicate the highest values of amplitude based on comparison between different stimuli and channels for a specific frequency.

\begin{tabular}{cccccccccc}
\hline \multirow{2}{*}{ Freq. } & Ch. & \multicolumn{9}{c}{ Group 1 } & \multicolumn{4}{c}{ Group 2 } \\
\cline { 3 - 10 } & & Red & Green & Blue & Yellow & Red & Green & Blue & Yellow \\
\hline $8 \mathrm{~Hz}$ & $\mathrm{O} 1$ & 0.32 & 0.44 & 0.37 & 0.48 & 0.37 & 0.36 & 0.30 & 0.30 \\
& $\mathrm{O} 2$ & 0.32 & 0.27 & 0.32 & 0.33 & 0.46 & 0.36 & 0.37 & 0.34 \\
& $\mathrm{Oz}$ & 0.36 & 0.43 & 0.49 & 0.47 & 0.38 & 0.37 & 0.36 & 0.42 \\
$11 \mathrm{~Hz}$ & $\mathrm{O} 1$ & 0.37 & 0.40 & 0.37 & 0.39 & 0.42 & 0.44 & 0.31 & 0.55 \\
& $\mathrm{O} 2$ & 0.34 & 0.25 & 0.32 & 0.29 & 0.56 & 0.34 & 0.47 & 0.46 \\
& $\mathrm{Oz}$ & 0.48 & 0.40 & 0.40 & 0.50 & 0.70 & 0.42 & 0.46 & 0.68 \\
$13 \mathrm{~Hz}$ & $\mathrm{O} 1$ & 0.56 & 0.35 & 0.31 & 0.37 & 0.70 & 0.49 & 0.35 & 0.59 \\
& $\mathrm{O} 2$ & 0.49 & 0.34 & 0.33 & 0.32 & 0.83 & 0.47 & 0.40 & 0.77 \\
& $\mathrm{Oz}$ & 0.75 & 0.43 & 0.42 & 0.54 & 1.00 & 0.65 & 0.55 & 0.86 \\
& $\mathrm{O} 15 \mathrm{~Hz}$ & 0.55 & 0.28 & 0.24 & 0.30 & 0.50 & 0.39 & 0.33 & 0.34 \\
& $\mathrm{O} 2$ & 0.36 & 0.25 & 0.26 & 0.29 & 0.70 & 0.41 & 0.34 & 0.38 \\
& $\mathrm{Oz}$ & 0.64 & 0.33 & 0.31 & 0.39 & 0.78 & 0.62 & 0.54 & 0.53 \\
\hline
\end{tabular}

Table 2. Score provided by volunteers about the level of comfort regarding the colored stimuli (7-point scale).

\begin{tabular}{cccccccccc}
\hline Group 1 & Red & Green & Blue & Yellow & Group 2 & Red & Green & Blue & Yellow \\
\hline s1 & 4 & 5 & 3 & 4 & $\mathrm{~s} 11$ & 6 & 2 & 7 & 3 \\
s2 & 2 & 5 & 6 & 3 & $\mathrm{~s} 12$ & 4 & 6 & 6 & 4 \\
s3 & 4 & 6 & 3 & 5 & $\mathrm{~s} 13$ & 2 & 5 & 6 & 4 \\
s4 & 3 & 6 & 4 & 3 & $\mathrm{~s} 14$ & 4 & 5 & 7 & 4 \\
s5 & 4 & 6 & 5 & 4 & $\mathrm{~s} 15$ & 4 & 6 & 7 & 3 \\
s6 & 3 & 5 & 4 & 4 & $\mathrm{~s} 16$ & 2 & 6 & 5 & 6 \\
s7 & 4 & 6 & 3 & 5 & $\mathrm{~s} 17$ & 5 & 6 & 3 & 5 \\
s8 & 2 & 5 & 5 & 3 & $\mathrm{~s} 18$ & 4 & 5 & 7 & 4 \\
s9 & 3 & 5 & 4 & 6 & $\mathrm{~s} 19$ & 3 & 5 & 5 & 3 \\
s10 & 4 & 7 & 3 & 4 & $\mathrm{~s} 20$ & 4 & 5 & 7 & 3 \\
Average & 3.30 & 5.60 & 4.00 & 4.10 & Average & 3.8 & 5.10 & 6.00 & 3.90 \\
SD & 0.82 & 0.70 & 1.05 & 0.99 & SD & 1.23 & 1.20 & 1.33 & 0.99 \\
\hline
\end{tabular}




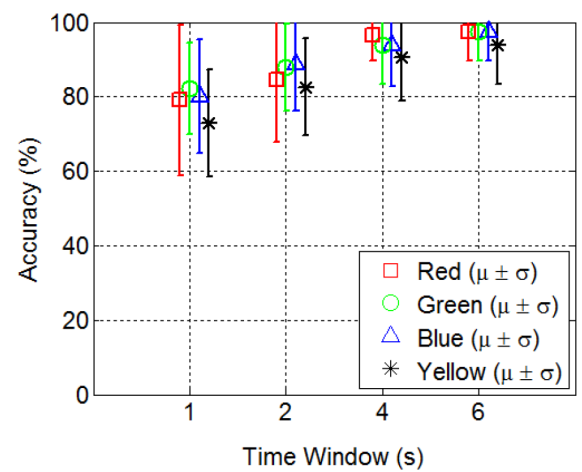

(a) - Group $1-8 \mathrm{~Hz}$

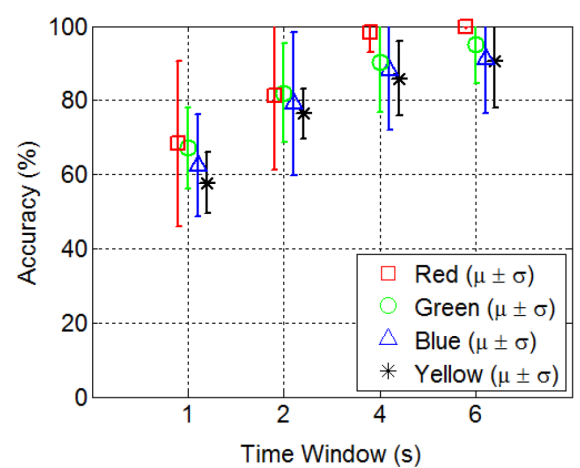

(b) - Group $1-11 \mathrm{~Hz}$

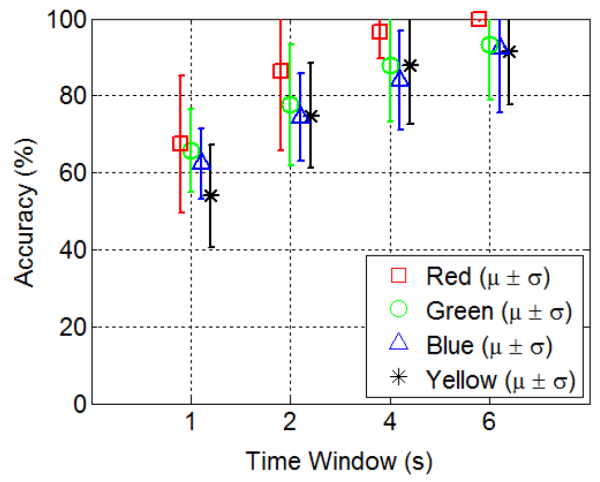

(c) - Group $1-13 \mathrm{~Hz}$

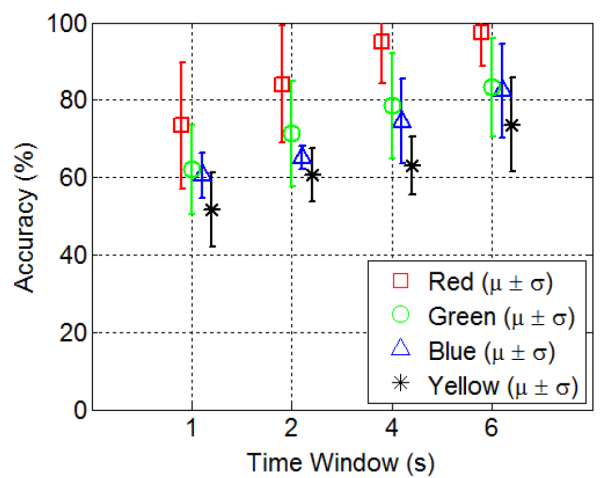

(d) - Group $1-15 \mathrm{~Hz}$

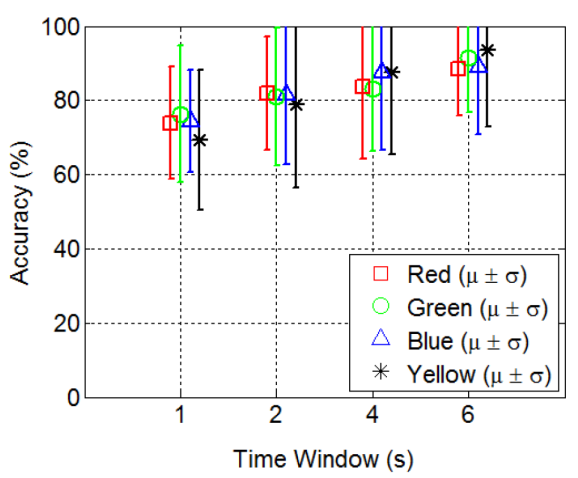

(e) - Group $2-8 \mathrm{~Hz}$

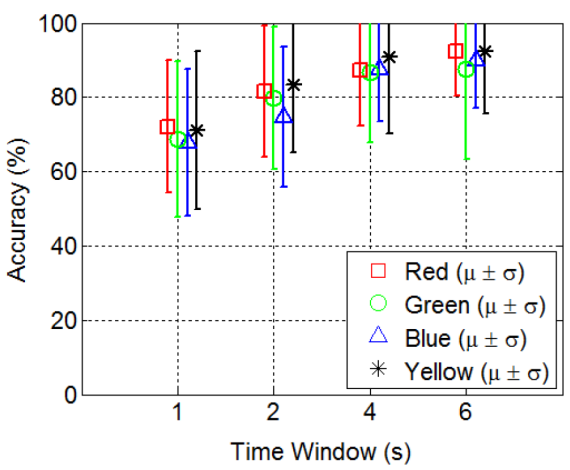

(f) - Group $2-11 \mathrm{~Hz}$

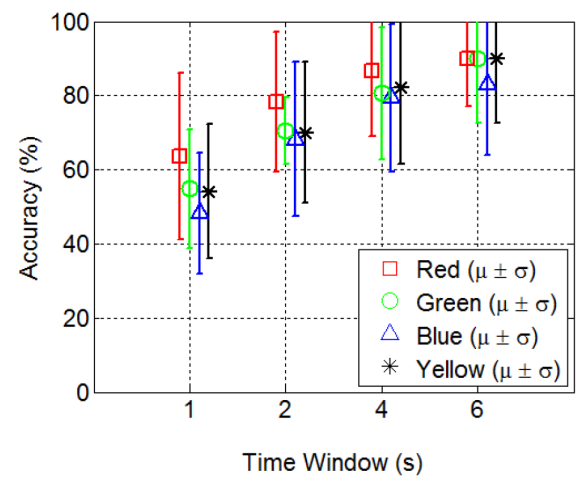

(g) - Group $2-13 \mathrm{~Hz}$

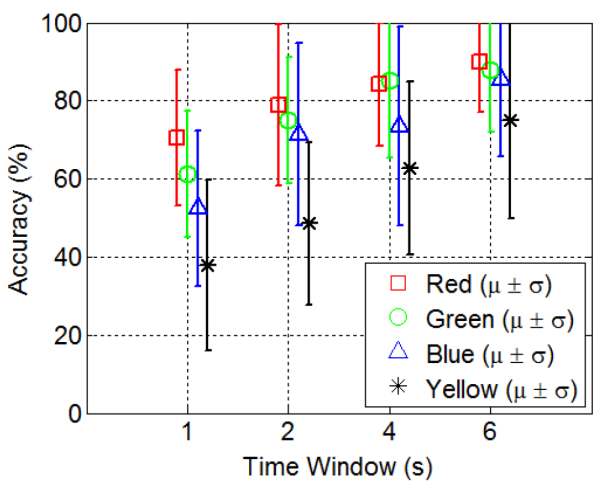

(h) - Group $2-15 \mathrm{~Hz}$

Figure 3. Hz. Average SSVEP recognition accuracy for each color using MSI method with different time windows and frequencies for Group 2: (e) for $8 \mathrm{~Hz}$, (f) for $11 \mathrm{~Hz}$, (g) for $13 \mathrm{~Hz}$, and (h) for $15 \mathrm{~Hz}$. 


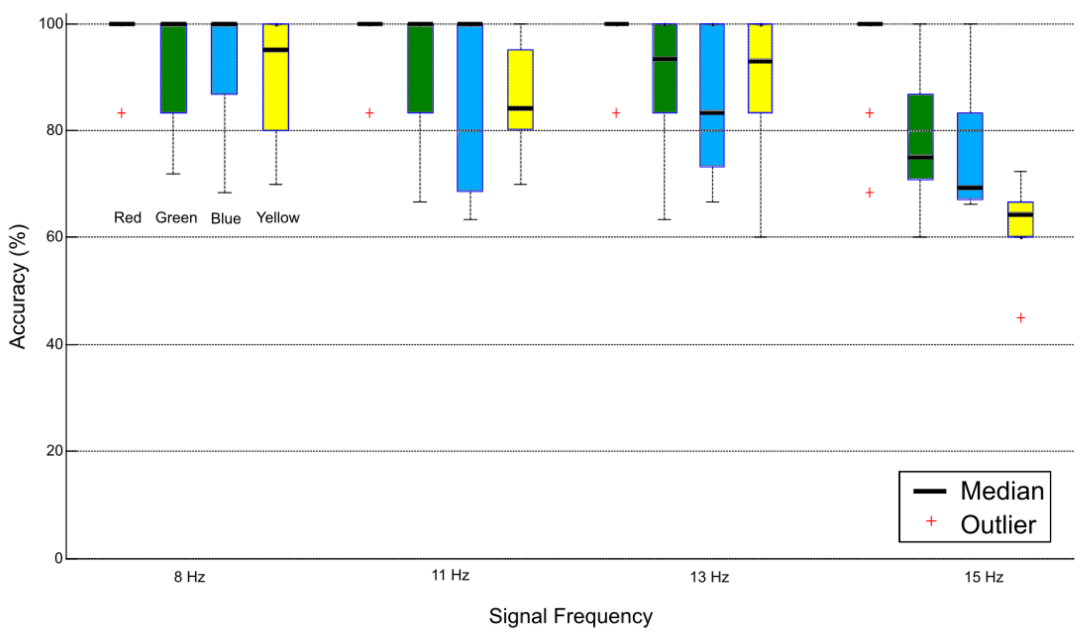

(a)

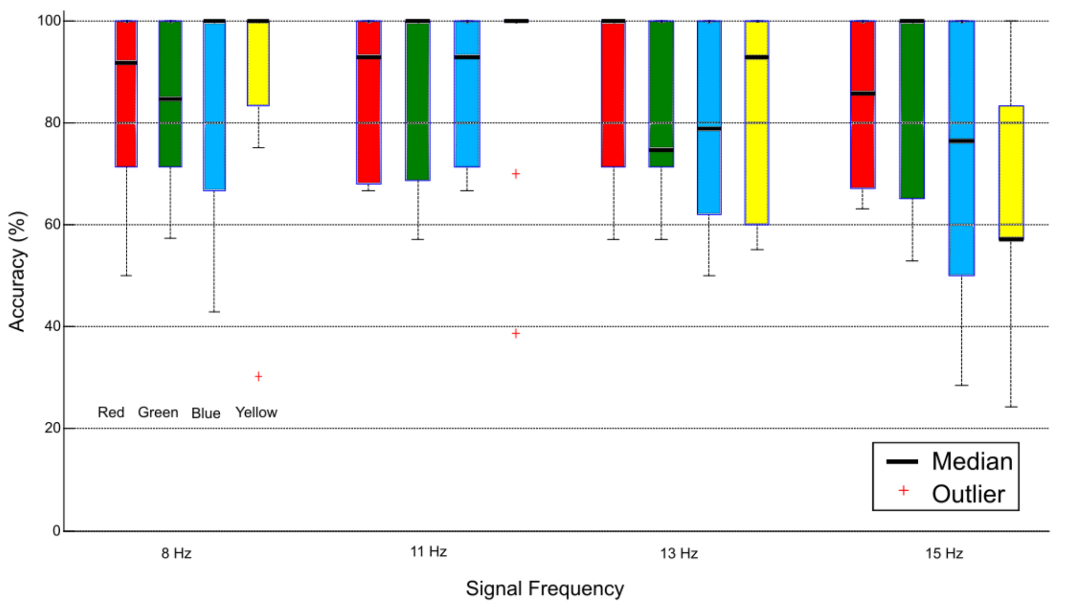

(b)

Figure 4. Median, quartiles and outliers of classification scores according color with time windows of $4 \mathrm{~s}$ corresponding to the different target frequencies $(8,11,13$ and $15 \mathrm{~Hz}$ ), obtained through MSI method for: (a) Group 1 and; (b) Group 2.

frequencies stimuli $(8,11,13$ and $15 \mathrm{~Hz})$ derived from the data of Group 1 and 2 with TW of $4 \mathrm{~s}$ using the MSI method. A statistical analysis was also implemented using Friedman's test with the purpose of finding out any effect between colors and frequencies. Thus, the frequencies were distributed in pairs for their analysis both for the ordered and random stimulation: (i) $8 \mathrm{~Hz}$ and $11 \mathrm{~Hz}$ vs colors; (ii) $8 \mathrm{~Hz}$ and $13 \mathrm{~Hz}$ vs colors; (iii) $8 \mathrm{~Hz}$ and $15 \mathrm{~Hz}$ vs colors; (iv) $11 \mathrm{~Hz}$ and $13 \mathrm{~Hz}$ vs colors; (v) $11 \mathrm{~Hz}$ and $15 \mathrm{~Hz}$ vs colors; (vi) $13 \mathrm{~Hz}$ and $15 \mathrm{~Hz}$ vs colors. The statistical analysis results showed that for Group 1: (i) $p=0.0363$, (ii) $p=0.1534$, (iii) $p=2.0765 \times 10-4$, (iv) $p=0.0142$, (v) $p=5.3185 \times 10-6$ and (vi) $p=1.2905 \times 10-4$. The combinations (i), (iii), (iv), (v) and (vi) were statistically significant using alpha $=0.05$. On the other hand, for Group 2: (i) $p=0.5589$, (ii) $p=0.9126$, (iii) $p=0.62$, (iv) $p=0.8457$, (v) $\mathrm{p}=0.6049$, (vi) $\mathrm{p}=0.1607$ were not statistically significant.

The ITR was calculated from the values of precision rate and compared with comfort values of each color stimuli. Comparative graphics for ITR versus comfort are shown in Figure 5. A diagonal line is shown in the graph, which was determined by two points: a starting point (coordinates indicating minimum level of comfort and minimum ITR) and an end point (maximum level of comfort and maximum ITR achieved when it gets $100 \%$ of accuracy). The values of the axis of ITR vary between a minimum and a maximum of 0 to $30 \mathrm{bits} / \mathrm{min}$, respectively. For each $5 \mathrm{bits} / \mathrm{min}$, there is one level of comfort associated.

The decision for the best choice of color stimuli was taken according to the following rules: 
- Each point of the graph represented a condition, involving data of ITR and comfort.

- The best choice of color was determined by the following: the point that is closer to the coordinate of maximum ITR and maximum comfort (upper right) is taken as the first best option, while the following closer ones define the order of the others remaining.

Figure 5 shows the comparison between the parameters of ITR versus comfort for TW of $4 \mathrm{~s}$. The graphics were distributed in stimulus frequency for both groups of volunteers. Moreover, Table 3
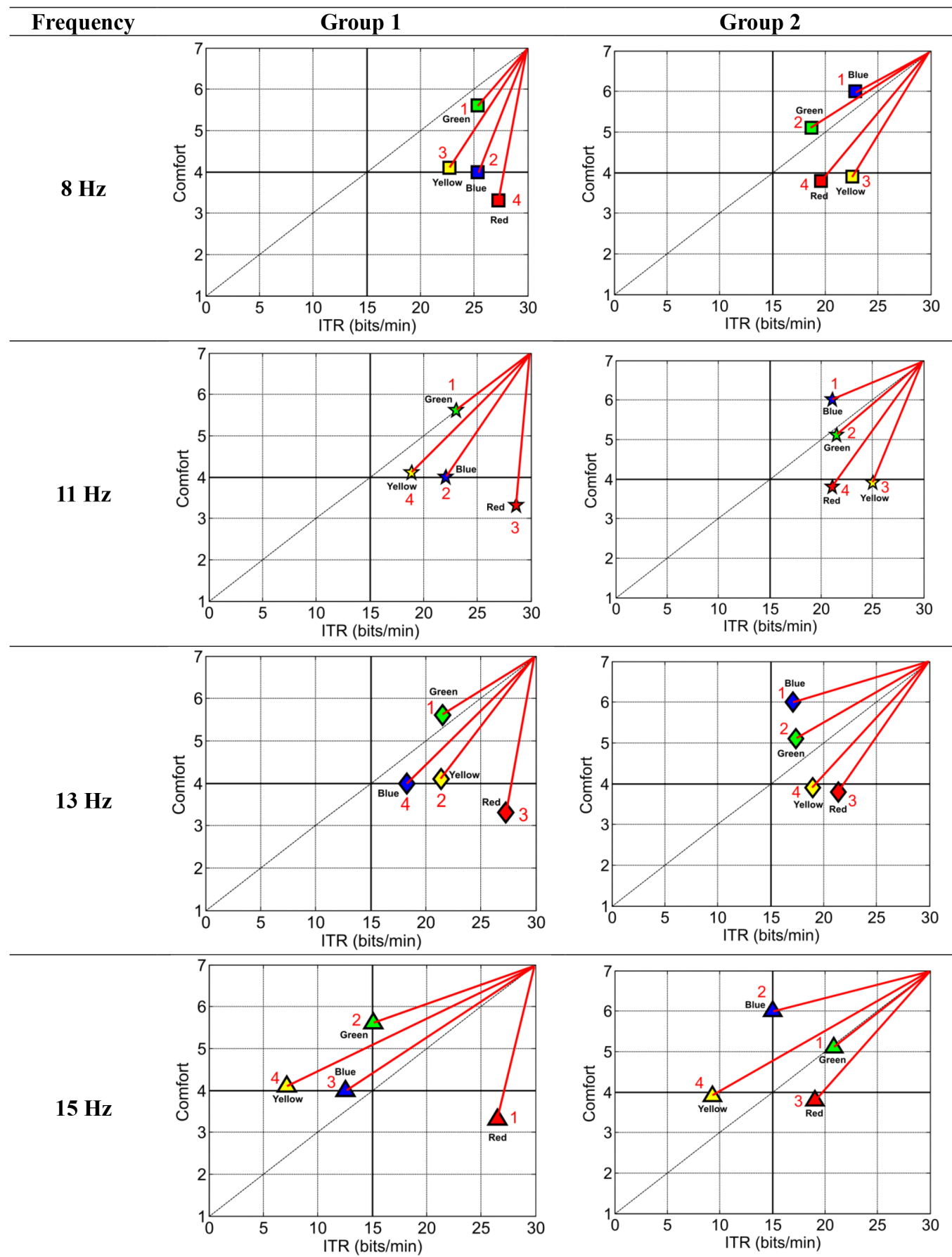

Figure 5. Plotting of average ITR versus average Comfort for color stimuli and different frequencies for volunteers of Group 1 and Group 2. In addition, the order of choice of colors is also shown. 
shows a summary of the values obtained in ITR and the choice of the best colors based on Figure 5. Note that these choices were based on levels of comfort and were called as first and second options.

In addition to results shown in Figure 5, the whole data were analyzed together. The data were grouped according to the color in spite of groups or frequencies used. Thus, centroids were calculated since the coordinate values of each condition are known (see Figure 6), and a single cluster is assigned for each color. The centroid of the cluster is the center of the circle indicated with a mark $\mathrm{x}$.

\section{Discussion}

According to Figure 2, the amplitude of the SSVEP response is affected by both frequency and color of the stimuli, confirming the studies initially performed by Regan (1966) and Drew et al. (2001).

In our study, the red color obtained the highest peaks of SSVEP amplitude in the majority of cases (see Table 1). An interesting fact to highlight is that the fundamental frequency of each color reached the highest peak of amplitude, which is confirmed by the

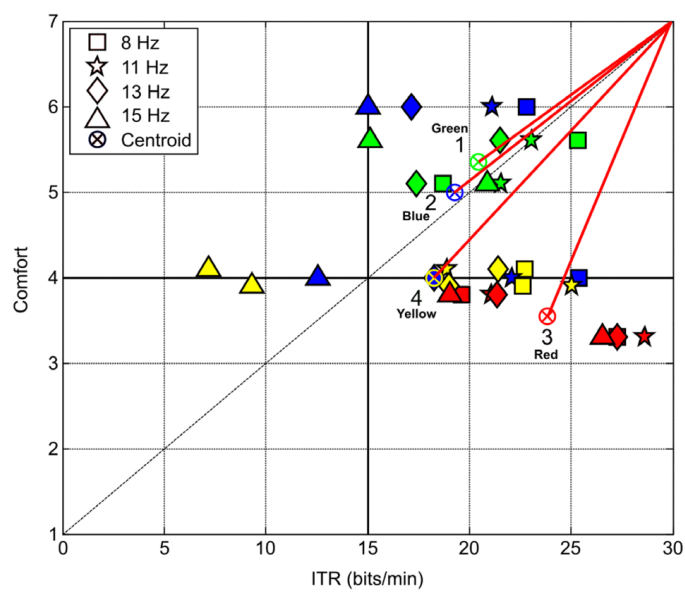

Figure 6. Scatter plot for all cases analyzed, clustered by color, using k-means method indicating its respective centroids. literature (Herrmann, 2001). Also the frequencies of 13 and $15 \mathrm{~Hz}$ reached the highest peaks of amplitude response for both groups, which is confirmed by studies described in the curve of Wang et al. (2006), where the middle frequencies (13 and $15 \mathrm{~Hz}$ ) exceeded in magnitude the low frequencies $(8$ and $11 \mathrm{~Hz})$.

On the other hand, Figure 3 showed that the SSVEP recognition accuracy is gradually enhanced with the increase of TW for all frequencies and groups of volunteers. This is widely known since SSVEP signals in larger time-windows yield better recognition of the visual evoked potentials (Zhang et al., 2014). According to the results of classification from Figure 3, a similar behavior in the results between group 1 and 2 can be appreciable. However, in the results of group 2 , a remarkable drop in the accuracy rate compared to its similar in the group 1 was observed. This fact may be related to visual accommodation when color stimuli of random way is presented, which is unlike of group 1 where the subjects previously knew the sequence of colors and frequencies. This aspect can be associated with the responses between color-sensitive cone pigments. Thus, that stabilization process could elicit delays in the modulation of attention. Modulation in attention is an important parameter for the increase of the precision in the recognition of visual evoked potentials. Despite of subjects having performed different protocols, in both groups the tendency in high accuracy terms is attributed for the red and green colors and frequencies of $8 \mathrm{~Hz}$. These evidences could help the development of accurate $\mathrm{BCIs}$ with frequencies very close to the beginning of alpha band, as in $8 \mathrm{~Hz}$, and also using red or green colored stimuli.

In Figure 4, accuracy rates using a box plot for different analyzed frequencies using a TW of $4 \mathrm{~s}$ are shown. There, the median, quartiles and outliers were presented for better appreciation of the distribution of our results. The success rate in 8 and $11 \mathrm{~Hz}$ achieved the highest results, which is contrary to the results of amplitude SSVEP presented in Figure 2, in which these frequencies have low amplitude. In addition,

Table 3. Average ITR (bits/min) for TW of $4 \mathrm{~s}$ and preferred colors by groups 1 and 2, according to color and frequency, respectively.

\begin{tabular}{|c|c|c|c|c|c|c|}
\hline Groups & $\begin{array}{c}\text { Frequency } \\
\qquad[\mathrm{Hz}]\end{array}$ & $\begin{array}{c}\text { Red ITR } \\
\text { [bits/min] }\end{array}$ & $\begin{array}{c}\text { Green ITR } \\
\text { [bits/min] }\end{array}$ & $\begin{array}{l}\text { Blue ITR } \\
\text { [bits/min] }\end{array}$ & $\begin{array}{c}\text { Yellow ITR } \\
\text { [bits/min] }\end{array}$ & $\begin{array}{l}\text { Better choices } \\
\text { (first choice / second choice) }\end{array}$ \\
\hline \multirow[t]{4}{*}{ Group 1} & 8 & 27.25 & 25.31 & 25.36 & 22.71 & Green/Blue \\
\hline & 11 & 28.63 & 23.05 & 22.07 & 18.89 & Green/Blue \\
\hline & 13 & 27.25 & 21.50 & 18.27 & 21.40 & Green/Yellow \\
\hline & 15 & 26.52 & 15.11 & 12.55 & 7.17 & Red/Green \\
\hline \multirow[t]{4}{*}{ Group 2} & 8 & 19.62 & 18.68 & 22.83 & 22.60 & Blue/Green \\
\hline & 11 & 21.09 & 21.54 & 21.10 & 25.06 & Blue/Green \\
\hline & 13 & 21.38 & 17.40 & 17.13 & 18.99 & Blue/Green \\
\hline & 15 & 19.04 & 20.86 & 15.01 & 9.30 & Green/Blue \\
\hline
\end{tabular}


an opposite situation occurs with the frequencies of 11 and $13 \mathrm{~Hz}$. These results would confirm some evidences about the fact that SSVEP response amplitude would not be decisive in the recognition rate, for the frequencies used, such as demonstrated in Kelly et al. (2005b). In their studies the frequencies in alpha band improve the recognition of SSVEPs during the modulation attentional. Regarding to the colors, it is remarkable to observe that the red color achieved the highest median values followed by green, blue and yellow in most cases for both group 1 and 2. In Group 1 and 2, a low accuracy rate in $15 \mathrm{~Hz}$ for yellow color was observed, which implies this color is not a good choice for development of a BCI.

According to Table 2, volunteers in groups 1 and 2 agree that the red color turned out to be quite uncomfortable as an option for visual stimulation. Moreover, the green color was chosen on average by group 1, and the blue by group 2, which were considered of best comfort for them. However, the blue (and yellow) obtained an intermediate opinion from group 1 with a standard deviation of 1 .

According to results of ITR from Table 3 , the red color got the highest values (between $25.52-28.63 \mathrm{bits} / \mathrm{min}$ ) for group 1. For group 2, red color reached the highest ITR only at $13 \mathrm{~Hz}$.

Based on our results, we observed that the red color provides, in most frequencies, both highest rates of accuracy and ITR for detection of SSVEP. These results confirm the studies of W. D. Wright (Gregory, 1997) and Regan (1966) about the response of the red color in the human eye. However, the red color, despite of the excellent results, showed to be the less comfortable to be used in a BCI. Moreover, the red color can be dangerous in combination with other colors (Drew et al., 2001) and, in low chromatic luminance, this color can induce epileptic responses (Ebersole and Pedley, 2002). This fact is confirmed by Rubboli et al. (2004), where red flicker could induce a Generalized Photoparoxysmal Response (PPR) having a relation to epilepsy and visually induced seizures. For these reasons, its use requires some care.

Additionally, in research on color and selective attention in the literature, the red color has shown to receive an attentional advantage (Elliot et al., 2014). In this aspect, researchers (Buechner et al., 2014; Elliot et al., 2014; Elliot, 2015; Lindsey et al., 2010; Pomerleau et al., 2014) agree that participants' visual search times were faster for desaturated red (relative to several other colored) targets.

On the other hand, research on color and alertness has shown that blue light increases subjective alertness and performance on attention-based tasks (Chellappa et al., 2011). This aspect is quite related to the fact that the attention is a powerful means of increasing the visual components of SSVEP and therefore increases the accuracy rate (Morgan et al., 1996).

In this present work, a study never before reported was performed by assessing twenty volunteers (none of them knew the protocol or had contact with a BCI), distributed into two different protocols. In this study, an analysis of performance of colored stimuli for SSVEP response using different flickering frequencies was performed. This is the first study published related to effects on the amplitude response and accuracy rate from visual stimuli of colored LEDs. In addition, subjective opinions from volunteers about aspects related to comfort were taken in account. The MSI method was implemented for feature extraction, where the SSVEP recognition uses pre-constructed sine-cosine waves as reference signals without information from training data.

For our results, although red color is clearly special and has garnered the majority of research attention, conceptually, blue and green seem reasonable candidates for use in SSVEP-BCI, as both have positive links and have been shown to be associated with positive content [blue, e.g., openness, peace (Mehta and Zhu, 2009); green, e.g., calmness, success (Moller et al., 2009)]. This fact is confirmed in Table 3 and Figure 5, which agree with studies of (Elliot, 2015; Mehta and Zhu, 2009; Moller et al., 2009), where green and blue got the highest nominations. The term "nomination" was attributed as number of times in which a kind of color is elected.

However, taking into account the large number of times nominated in our studies ( 5 out of 8 , only as first choice), the green color is suggested in both groups as the first best choice for a comfortable, safe and accurate SSVEP-BCI. The red color was disregard of our nominations due to its low level of comfort and its trends to evoke epileptic responses. The results from Figure 6 confirm that green color is the most suitable option for visual stimulation, where the rule of selection of the best color was applied to each resulting centroid. Finally, the order of choice was the following: green, blue and yellow. It is worth to comment that these results were obtained in laboratory conditions and using a black background. An interesting future work could evaluate the SSVEP recognition using different colors of background.

\section{Acknowledgements}

The first author thanks CAPES/Brazil for the scholarship granted and also acknowledges the collaboration of the volunteers who helped the realization of this research. 


\section{References}

Bieger J, Garcia-Molina G, Zhu D. Effects of stimulation properties in Steady-State Visual Evoked Potential based Brain-Computer Interfaces. In: Proceedings of 32nd Annual International Conference of the IEEE Engineering in Medicine and Biology Society; 2010 Aug 31-Sep 04; Buenos Aires, Argentina. Engineering in Medicine and Biology Society; 2010. p. $3345-8$

Bieger J, Garcia-Molina G. Light stimulation properties to influence brain activity: a brain-computer interface application [internet]. Philips Research; 2010 Oct [cited 2015 Feb 26]; 7-9. Available from: http://repository.tudelft.nl/view/philips/ uuid\%3A617bbd89-8ab8-49da-9fc8-f6c7803724c9/.

Buechner VL, Maier MA, Lichtenfeld S, Schwarz S. Red - take a closer look. PLoS One. 2014; 9(9):e108111. http:// dx.doi.org/10.1371/journal.pone.0108111. PMid:25254380

Cao T, Wan F, Peng UM, Pui-In M, Mang IV, Yong H. Flashing color on the performance of SSVEP-based Brain-Computer Interfaces. In: International Conference of the IEEE EMBS; 2012; San Diego, CA. IEEE; 2012. p. 1819-22. http://dx.doi.org/10.1109/EMBC.2012.6346304.

Carmeli C, Knyazeva MG, Innocenti GM, De Feo O. Assessment of EEG synchronization based on state-space analysis. NeuroImage. 2005; 25(2):339-54. http://dx.doi. org/10.1016/j.neuroimage.2004.11.049. PMid:15784413.

Chellappa SL, Steiner R, Blattner P, Oelhafen P, Götz $\mathrm{T}$, Cajochen $\mathrm{C}$. Non-visual effects of light on melatonin, alertness and cognitive performance: can blue-enriched light keep us alert? PLoS One. 2011; 6(1):e16429. http:// dx.doi.org/10.1371/journal.pone.0016429. PMid:21298068.

Di Russo F, Pitzalis S, Aprile T, Spitoni G, Patria F, Stella A, Spinelli D, Hillyard SA. Spatiotemporal analysis of the cortical sources of the steady-state visual evoked potential. Human Brain Mapping. 2007; 28(4):323-34. http://dx.doi. org/10.1002/hbm.20276. PMid:16779799.

Drew P, Sayres R, Watanabe K, Shimojo S. Pupillary response to chromatic flicker. Experimental Brain Research. 2001; 136(2):256-62. http://dx.doi.org/10.1007/s002210000605. PMid:11206288.

Ebersole JS, Pedley TA. Current practice of clinical electroencephalography. 3rd ed. Philadelphia: Lippincott Williams \& Wilkins; 2002.

Elliot AJ. Color and psychological functioning: a review of theoretical and empirical work. Frontiers in Psychology. 2015; 6(368):368. http://dx.doi.org/10.3389/fpsyg.2015.00368. PMid:25883578.

Elliot AJ, Fairchild MD, Franklin A. Handbook of color psychology. Cambridge: Cambridge University Press; 2014.

Fisher RS, Harding G, Erba G, Barkley GL, Wilkins A. Photic- and pattern-induced seizures: a review for the epilepsy foundation of America working group. Epilepsia. 2005; 46(9):1426-41. http://dx.doi.org/10.1111/j.15281167.2005.31405.x. PMid:16146439.

Gregory R. Eye and brain the psychology of seeing. Princeton: Princeton University Press; 1997.
Herrmann CS. Human EEG responses to 1-100 Hz flicker: resonance phenomena in visual cortex and their potential correlation to cognitive phenomena. Experimental Brain Research. 2001; 137(3-4):346-53. http://dx.doi.org/10.1007/ s002210100682. PMid:11355381.

Kelly SP, Lalor EC, Finucane C, McDarby G, Reilly RB. Visual spatial attention control in an independent braincomputer interface. IEEE Transactions on Biomedical Engineering. 2005a; 52(9):1588-96. http://dx.doi.org/10.1109/ TBME.2005.851510. PMid:16189972.

Kelly SP, Lalor EC, Reilly RB, Foxe JJ. Independent Brain Computer Interface control using visual spatial attentiondependent modulations of parieto-occipital alpha. In: Proceedings of the 2nd International IEEE EMBS Conference on Neural Engineering; 2005; Arlington, VA. IEEE; 2005 b. p. 667-70. http://dx.doi.org/10.1109/CNE.2005.1419713.

Krolak-Salmon P, Hénaff MA, Tallon-Baudry C, Yvert B, Guénot M, Vighetto A, Mauguière F, Bertrand O. Human lateral geniculate nucleus and visual cortex respond to screen flicker. Annals of Neurology. 2003; 53(1):73-80. http://dx.doi.org/10.1002/ana.10403. PMid:12509850.

Lindsey DT, Brown AM, Reijnen E, Rich AN, Kuzmova YI, Wolfe JM. Color channels, not color appearance or color categories, guide visual search for desaturated color targets. Psychological Science. 2010; 21(9):1208-14. http:// dx.doi.org/10.1177/0956797610379861. PMid:20713637.

McFarland DJ, McCane LM, David SV, Wolpaw JR. Spatial filter selection for EEG-based communication. Electroencephalography and Clinical Neurophysiology. 1997; 103(3):386-94. http://dx.doi.org/10.1016/S00134694(97)00022-2. PMid:9305287.

Mehta R, Zhu RJ. Blue or red? Exploring the effect of color on cognitive task performances. Science. 2009; 323(5918):1226-9. http://dx.doi.org/10.1126/science.1169144. PMid:19197022.

Moller AC, Elliot AJ, Maier MA. Basic hue-meaning associations. Emotion. 2009; 9(6):898-902. http://dx.doi. org/10.1037/a0017811. PMid:20001133.

Morgan ST, Hansen JC, Hillyard SA. Selective attention to stimulus location modulates the steady-state visual evoked potential. Proceedings of the National Academy of Sciences of the United States of America. 1996; 93(10):4770-4. http://dx.doi.org/10.1073/pnas.93.10.4770. PMid:8643478.

Pastor MA, Artieda J, Arbizu J, Valencia M, Masdeu JC. Human cerebral activation during steady-state visualevoked responses. The Journal of Neuroscience. 2003; 23(37):11621-7. PMid:14684864.

Pomerleau VJ, Fortier-Gauthier U, Corriveau I, Dell'Acqua $\mathrm{R}$, Jolicœur P. Colour-specific differences in attentional deployment for equiluminant pop-out colours: evidence from lateralised potentials. International Journal of Psychophysiology. 2014; 91(3):194-205. http://dx.doi. org/10.1016/j.ijpsycho.2013.10.016. PMid:24188915.

Regan D. An effect of stimulus colour on average steady-state potentials evoked in man. Nature. 1966; 210(5040):1056-7. http://dx.doi.org/10.1038/2101056a0. PMid:5914904. 
Rubboli G, Parra J, Seri S, Takahashi T, Thomas P. EEG diagnostic procedures and special investigations in the assessment of photosensitivity. Epilepsia. 2004; 45(1 Suppl 1):35-9. http://dx.doi.org/10.1111/j.0013-9580.2004.451002.x. PMid:14706044.

Sammer G, Blecker C, Gebhardt H, Kirsch P, Stark R, Vaitl D. Acquisition of typical EEG waveforms during fMRI: SSVEP, LRP, and frontal theta. NeuroImage. 2005; 24(4):101224. http://dx.doi.org/10.1016/j.neuroimage.2004.10.026. PMid: 15670678 .

Tello RJMG, Muller SMT, Bastos TF Fo, Ferreira A. A comparison of techniques and technologies for SSVEP classification. In: IEEE-ISSNIP Biosignals and Biorobotics Conference: Biosignals and Robotics for Better and Safer Living (BRC); 2014; Salvador. IEEE; 2014a. p. 1-6. http:// dx.doi.org/10.1109/BRC.2014.6880956.

Tello RJMG, Muller SMT, Bastos TF Fo, Ferreira A. Comparison of new techniques based on EMD for control of a SSVEP-BCI. In: IEEE 23rd International Symposium on Industrial Electronics (ISIE); 2014; Istanbul. IEEE; 2014b. p. 992-7. http://dx.doi.org/10.1109/ISIE.2014.6864747.

Tello RJMG, Muller SMT, Bastos TF Fo, Ferreira A. Comparison between wire and wireless EEG acquisition systems based on SSVEP in an independent-BCI. In: 36th Annual International Conference of the IEEE Engineering in Medicine and Biology Society (EMBC'14). 2014c. p. 22-5. http://dx.doi.org/10.1109/EMBC.2014.6943519.

Tello RG, Pant JK, Müller SM, Krishnan S, Bastos TF Fo. An evaluation of performance for an independent SSVEP-BCI based on compressive sensing system. In: Jaffray DA. World Congress on Medical Physics and Biomedical Engineering; 2015 June 7-12; Toronto, Canada. Springer International Publishing; 2015. p. 982-5. (IFMBE Proceedings, 51). http:// dx.doi.org/10.1007/978-3-319-19387-8_239.

Vialatte FB, Maurice M, Dauwels J, Cichocki A. Steady-state visually evoked potentials: focus on essential paradigms and future perspectives. Progress in Neurobiology. 2010; 90(4):418-38. http://dx.doi.org/10.1016/j.pneurobio.2009.11.005. PMid:19963032.

Wang Y, Wang R, Gao X, Hong B, Gao S. A practical VEP based brain-computer interface. IEEE Transactions on Neural Systems and Rehabilitation Engineering. 2006; 14(2):234-40. http://dx.doi.org/10.1109/TNSRE.2006.875576.

Wu Z, Lai Y, Xia Y, Wu D, Yao D. Stimulator selection in SSVEP-based BCI. Medical Engineering \& Physics. 2008; 30(8):1079-88. http://dx.doi.org/10.1016/j. medengphy.2008.01.004. PMid:18316226.

Zhang Y, Wang R, Hong B, Gao X, Gao S. Source estimation of contrast-related perception based on frequency-tagged binocular rivalry. In: EMBS ‘ 06.28 th Annual International Conference of Engineering in Medicine and Biology Society; 2006; New York, NY. IEEE; 2006. p. 1177-80. http://dx.doi. org/10.1109/IEMBS.2006.260094.

Zhang Y, Xu P, Cheng K, Yao D. Multivariate synchronization index for frequency recognition of SSVEP-based braincomputer interface. Journal of Neuroscience Methods. 2014; 221(1):32-40. http://dx.doi.org/10.1016/j.jneumeth.2013.07.018. PMid:23928153.

\section{Authors}

Richard Junior Manuel Godinez Tello ${ }^{1 *}$, Sandra Mara Torres Müller ${ }^{2}$, André Ferreira ${ }^{1}$, Teodiano Freire Bastos ${ }^{1}$ ${ }^{1}$ Programa de Pós-Graduação em Engenharia Elétrica - PPGEE, Universidade Federal do Espírito Santo - UFES, Av. Fernando Ferrari, 514, CEP 29075-910, Vitória, ES, Brazil. 